\title{
PEDILANTHUS CONNATUS (EUPHORBIACEAE), ESPECIE NUEVA Y LLAMATIVA DE JALISCO
}

\author{
Robert L. Dressler \\ Department of Natural Sciences \\ Florida Museum of Natural History \\ University of Florida \\ Gainesville, Florida 32611, E.U.A. \\ Charles M. Sacamano \\ Apartado 81A, Aeropuerto \\ Puerto Vallarta, Jalisco 48301, México
}

\section{RESUMEN}

Se describe Pedilanthus connatus del oeste de Jalisco, especie que parece ser cercana a $P$. coalcomanensis. Es de hábito arbustivo y rizomatoso, y parece producir flores durante todo el año cuando hay agua suficiente. Podría ser de interés como planta de adorno.

\section{ABSTRACT}

Pedilanthus connatus is described from western Jalisco. It appears to be a close ally of $P$. coalcomanensis. It is a slender, rhizomatous shrub and appears to flower throughout the year when moisture is sufficient. It may hold promise as a garden ornamental.

Pedilanthus es esencialmente un conjunto mexicano. Todas las especies se encuentrań en México, con excepción de la poco conocida $P$. millspaughii, descrita de Costa Rica. De los cinco grupos reconocidos por Dressler (1957), cuatro están representados en el oeste de México, y cada uno de estos grupos incluye una especie relativamente primitiva en el occidente mexicano, sugiriendo que el género puede haber evolucionado en esa región. Aquí se describe una especie nueva y distintiva del oeste de Jalisco.

Pedilanthus connatus Dressler et Sacamano, sp. nov. Fig. 1

Arbuscula erecta; foliis glabris, oblongo-obovatis, acutis vel breviter acuminatis; cymis terminalibus et axillaribus, bracteis late ovatis, puberulis; involucris puberulis, calcaribus quam involucris longioribus, lobis centralibus connatis; glandulis 4, globosooblongis; fructus seminaque ignota.

Arbusto erecto $0.5-2 \mathrm{~m}$ de alto, con raíz carnosa o rizoma horizontal carnoso; tallos delgados, los jóvenes 3-5 mm de diámetro, ligeramente pubérulos, frecuentemente rojizos, 


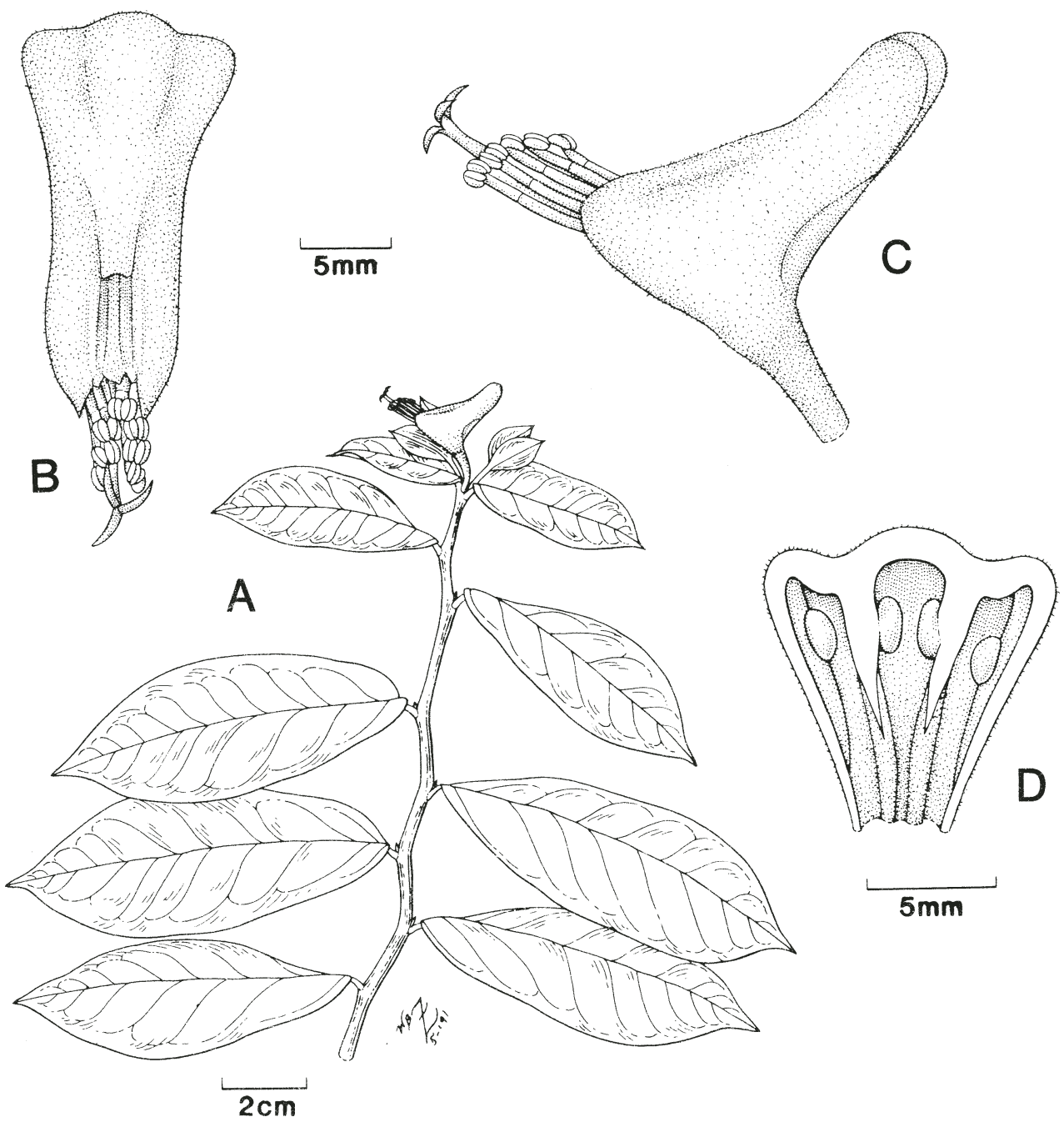

Fig. 1. Pedilanthus connatus. A. rama con inflorescenia joven; B. ciatio, vista dorsal; C. ciatio, vista lateral; D. vista dorsal del espolón, sección mostrando la cámara de glándulas. A, del holótipo; B-D, de material conservado en liquido. En la vista dorsal del ciatio (B) los lobulos principales del involucro son laterales (abajo), con los tres lobulos accesorios intermedios. En el espolón (arriba) los lóbulos están unidos hasta considerable altura; el lóbulo encima de los lobulos accesorios del involucro representa los ápices unidos de los lóbulos intermedios del espolón. 
tallos viejos hasta $2 \mathrm{~cm}$ de diámetro; hojas oblongo-obovadas, $7-10 \mathrm{~cm}$ de largo, 2.5-4.5 $\mathrm{cm}$ de ancho, base obtusa, ápice agudo hasta brevemente acuminado, hojas lampinas en el haz, ligeramente pubérulas en el envés en venas y bordes, vena central prominente, aquillada hacia la base, peciolo 3-4 mm de largo, pubérulo; estípulas castaño-rojizas, de apariencia glandular, aprox. $0.3 \mathrm{~mm}$ de diámetro; inflorescencia terminal y en las axilas superiores, de pocos a varios ciatios, ramificación dicasial o monocasial, entrenudos hasta $12 \mathrm{~mm}$ de largo, pubérulos, primer par de brácteas parecidas a las hojas, verdes o teñidas de rosado, 3-6 cm de largo, $1.5-3 \mathrm{~cm}$ de ancho, brácteas subsecuentes anchamente ovadas, hasta $15 \mathrm{~mm}$ de largo, $10 \mathrm{~mm}$ de ancho, rosadas, pubérulas, caducas; pedúnculos 8-11 mm de largo, pubérulos; ciatio rosado, pubérulo salvo en la cámara de glándulas, tubo involucral $12-13 \mathrm{~mm}$ de largo, lobulos principales deltoide-ovados, obtusos, ápices libres ventralmente por espacio de $3.4 \mathrm{~mm}$; l6bulos accesorios liguliformes, dorsalmente aquillados, más cortos que los lobulos principales, estrechamente obtusos, pubérulos, aprox. $1 \mathrm{~mm}$ de ancho, ápices libres por espacio de $2-3 \mathrm{~mm}$; espolón oblicuo al tubo involucral, $17-18 \mathrm{~mm}$ de largo, aprox. $12 \mathrm{~mm}$ de ancho en el ápice, truncado, ligeramente trilobado, lóbulos laterales del espolón lanceolado-liguliformes, su anchura disminuyendo hacia abajo hasta unirse con el tubo involucral, lobulos intermedios unidos, de aprox. 15 $\mathrm{mm}$ de largo desde el ápice del espolón, libres por espacio de 6-7 mm, lámina libre deltoideoblonga, el ápice ligeramente retuso, aprox. $4 \mathrm{~mm}$ de ancho en la base y $1.8 \mathrm{~mm}$ en el ápice, espolón dividido por dentro por 2 tabiques de 6-7 mm de largo, glándulas 4, las exteriores globoso-oblongas, aprox. $1.7 \mathrm{~mm}$ de largo, las interiores oblongas, aprox. $2 \mathrm{~mm}$ de largo; flores estaminadas numerosas, pedicelos 14-16 mm de largo, ligeramente pubérulos, filamentos aprox. $3 \mathrm{~mm}$ de largo, pubérulos; pedicelo pistilado grueso, pubérulo, 15-17 mm de largo, ovario aprox. $3 \mathrm{~mm}$ de largo, triquetro, con un cáliz basal rudimentario, estilo aprox. $7 \mathrm{~mm}$ de largo, divisiones estigmáticas 3, aprox. $1.5 \mathrm{~mm}$ de largo; cápsula y semillas desconocidas.

MEXICO: Jalisco, about $40 \mathrm{~km}$ SE of Puerto Vallarta and $50 \mathrm{~km}$ inland from Pacific ocean; rugged mountainous terrain at $3400 \mathrm{ft}$. elevation; erect, single-stemmed herb to $0.5 \mathrm{~m}$ tall; inflorescence terminal, of 1-5 cyathia which are clear medium pink; coll. July 10, 1987; prepared from greenhouse-grown plants, 7 April 1988, Charles Sacamano s.n. Holótipo (MO); otro espécimen visto: about $30 \mathrm{~km}$ SE of Puerto Vallarta, near road to mining village of Cuale; elevation $1100 \mathrm{~m}$; collected 17 August 1987; flowering material pressed, December 1988, C. Sacamano s. n. (GUADA).

Pedilanthus connatus parece estar estrechamente emparentada con $P$. coalcomanensis, con la cual comparte los lobulos intermedios del espolón unidos y el número y arreglo de las glándulas. Pedilanthus coalcomanensis es un árbol con el tubo involucral del ciatio más largo que el espolón, y la cápsula normalmente se desarrolla dentro del involucro. Pedilanthus connatus es un arbusto más bien delgado, el espolón es más largo que el tubo involucral y el pedicelo pistilado se prolonga, exponiendo la cápsula. Los lóbulos intermedios del espolón de $P$. connatus están unidos entre sl, y también están unidos con los lóbulos laterales en la mitad basal. El epíteto connatus se refiere al alto grado de unión entre los lóbulos del espolón.

Pedilanthus connatus se encuentra en el sotobosque debajo de Quercus en laderas empinadas y rocosas. Las plantas con un sistema vertical de ralces carnosas parecen 
ser individuos jóvenes originados de semilla. La mayorla de las plantas poseen un rizoma carnoso horizontal de 10 a $15 \mathrm{~cm}$ debajo de la superficie, con dos o más tallos aéreos originándose de cada rizoma. Hasta ahora el hábito rizomatoso sólo se conocia en Pedilanthus en especies áfilas y desérticas, como $P$. cymbiferus. La mayoría de las especies de Pedilanthus, y especialmente las especies de hojas grandes y tallos delgados, son plantas de "día corto", con su floración en los meses secos. Pedilanthus connatus se ha observado con flores en la localidad típica a fines de mayo y de julio a mediados de noviembre (en un año lluvioso). En cultivo esta especie florece casi constantemente si hay humedad adecuada. A causa de su tamaño moderado, su inflorescencia llamativa y su época larga de floración, esta especie puede ser de interés como planta de adorno en jardines $\theta$ invernaderos.

\section{AGRADECIMIENTOS}

Damos las gracias a la Dra. Wendy Zomlefer por la preparación del dibujo.

\section{LITERATURA CITADA}

Dressler, R. L. 1957. The genus Pedilanthus (Euphorbiaceae). Contr. Gray Herbarium 182: 1-188. 